\title{
Room temperature, continuous-wave coupled-cavity InAsP/InP photonic crystal laser with enhanced far-field emission directionality
}

\author{
Jingqing Huang, ${ }^{1,2, a)}$ Se-Heon Kim, ${ }^{1,2}$ Jonathan Gardner, ${ }^{1}$ Philippe Regreny, ${ }^{3}$ \\ Christian Seassal, ${ }^{3}$ Pablo Aitor Postigo, ${ }^{4}$ and Axel Scherer ${ }^{1,2}$ \\ ${ }^{1}$ Department of Electrical Engineering, California Institute of Technology, Pasadena, California 91125, USA \\ ${ }^{2}$ Kavli Nanoscience Institute, California Institute of Technology, Pasadena, California 91125, USA \\ ${ }^{3}$ Université de Lyon, Institut des Nanotechnologies de Lyon INL-UMR 5270, CNRS, Ecole Centrale de Lyon, \\ Avenue Guy de Collongue, Cedex F-69134, France

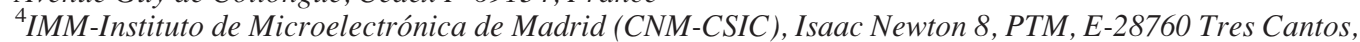 \\ Madrid, Spain
}

(Received 30 June 2011; accepted 12 August 2011; published online 1 September 2011)

\begin{abstract}
We demonstrate room temperature, continuous-wave lasing with enhanced far field emission directionality in coupled-cavity photonic crystal lasers, made with InAsP/InP quantum well material. These surface-emitting lasers can have a very low effective threshold power of $14.6 \mu \mathrm{W}$, with a linewidth of $60 \mathrm{pm}$, and $40 \%$ of the surface emitted power concentrated within a small divergence angle of $\pm 30^{\circ}$. (C) 2011 American Institute of Physics. [doi:10.1063/1.3633120]
\end{abstract}

Wavelength- and subwavelength-scale lasers are useful devices for on-chip optical signal processing, communication, and biochemical spectroscopy. ${ }^{1-3}$ Their small sizes bring the advantages of small on-chip footprint and potential of Purcell enhancement, which may lead to faster laser modulation and thresholdless lasing. ${ }^{4-6}$ Room temperature (RT), continuous-wave $(\mathrm{CW})$ operation is requisite for stable lasing characteristics and narrow linewidths and has been achieved with quantum dot, quantum wire, and quantum well (QW) materials using a variety of photonic crystal $(\mathrm{PhC})$ cavity designs. ${ }^{6-8}$ However, most of the wavelengthscale laser cavities are so far optimized for high quality factor $(Q)$ and small effective volume $\left(V_{\text {eff }}\right)$ in attempt to maximize the Purcell factor $F_{p}$, but are not particularly designed for efficient light extraction. Extraction methods such as evanescent coupling to a tapered microfiber ${ }^{9}$ and monolithic integration with a passive waveguide using wafer regrowth techniques ${ }^{10}$ have been demonstrated; lasers with highly directional surface emission can provide an alternative way to achieve efficient free-space light coupling. ${ }^{11}$ At the same time, coupled photonic crystal lasers have been studied to increase lasing power and differential quantum efficiency. ${ }^{12}$ Single mode operation can be achieved from coupled cavities even when they are individually multimode, as the constituent cavities act as mode filters for each other. ${ }^{13}$ To further investigate and improve on these issues, we have designed coupled photonic crystal cavities that exhibit single mode lasing and enhanced emission directionality. We show experimental measurements of fabricated devices and fit the measured data to laser rate equations to estimate the laser's threshold, spontaneous emission coupling factor $(\beta)$, as well as the $1.5 \mu \mathrm{m}$ wavelength emitting InAsP/InP QW material properties for future nanolaser designs in this not yet commonly used material system.

Our devices are formed in an InP slab with a thickness $d$ of $240 \mathrm{~nm}$ on top of a $1.16 \mu \mathrm{m}$ thick sacrificial InGaAs layer. The InP slab contains 4 InAsP QWs with the photolumines-

${ }^{\text {a)} E l e c t r o n i c ~ m a i l: ~ j i n g q i n g @ c a l t e c h . e d u . ~}$ cent emission peak at $1550 \mathrm{~nm}$. Cylindrical holes etched into the InP slab in a hexagonal lattice form the PhC. One missing air hole forms a simple H1 cavity, 3 or 5 missing air holes along the $\Gamma-\mathrm{K}$ direction form an L3 or L5 cavity, respectively. We laterally couple these cavities as shown in Fig. 1: Design A consists of L3-L5-L3 cavities and Design B consists of H1-L3-L5-L3-H1 cavities. The samples are fabricated using electron beam lithography on poly(methyl methacrylate) (PMMA) resist, followed by a chemically assisted ion beam etch (CAIBE) with $\mathrm{Ar} / \mathrm{Cl}_{2}$ to form the air holes and an oxygen plasma to remove the residual resist. Finally, the InGaAs layer is etched away in a dilute $\mathrm{H}_{2} \mathrm{CrO}_{4} / \mathrm{HF}$ mixture to suspend the $\mathrm{InP} / \mathrm{InAsP} \mathrm{PhC}$ slab. The best performing devices have a lattice period of $a=470 \mathrm{~nm}$ and air hole radius of $r=0.3 a$. Fig. 1(a) shows SEM images of finished devices.

The coupled-cavity lasers are measured in a photoluminescence set-up at room temperature, pumped continuously with a $\lambda_{p}=832 \mathrm{~nm}$ diode laser. The pump laser beam is focused to an approximately $4 \mu \mathrm{m}$ diameter spot on the sample surface using a $50 \times$ microscope objective. Emitted light from the devices is collected through the same objective and recorded using an optical spectrum analyzer (OSA). Finitedifference-time-domain (FDTD) simulations reveal that the coupled-cavities in Design A have several resonance modes

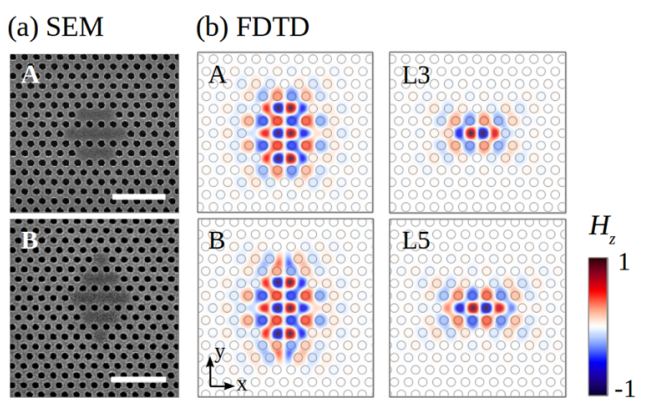

FIG. 1. (Color online) (a) SEM images of fabricated devices for Designs A and B. (b) Normalized $H_{z}$ field component of the lasing modes for L3, L5, and coupled-cavities. 
within the InP/InAsP QW material's gain spectrum, among which the highest $Q$ mode has a $Q$ of 3100 , while all other modes have $Q$ of a few hundreds to 1300 . In Design B, the highest $Q$ mode within the photoluminescence bandwidth is 1700 , all other modes have $Q$ 's of below 1000 . Only the highest $Q$ mode, whose mode profile is shown in Fig. 1(b), tends to achieve the lasing threshold condition, making our devices single-mode lasers, as is evidenced in measured lasing spectra shown in Fig. 2(a). CCD camera images of lasing devices are shown in insets. They represent vertically directed Poynting energy flux $P_{z}$ from the lasing mode at approximately one lasing wavelength above the devices' top surface. The corresponding FDTD simulations of this Poynting flux distribution, i.e., $\left(\omega_{l} / 2 \pi\right) \int_{0}^{2 \pi / \omega_{l}} P_{z}\left(x, y, z \approx \lambda_{l}\right) d t$ where $\omega_{l}$ is the angular frequency of the lasing mode, confirm the main features in the lobed patterns seen on CCD: Design A has two major side lobes and Design $\mathrm{B}$ has a bright center with four minor lobes.

Efficient free-space coupling requires the laser to have a directional far-field radiation pattern. The proposed schemes so far for directional emission from photonic crystal microcavity lasers involve fine modification of the air-holes in the vicinity of the main cavity. ${ }^{11,14}$ Here, we show that far-field directionality can be engineered by proper arrangement of coupled cavities to a level that is satisfactory for practical application without fine-tuning the $\mathrm{PhC}$ air-holes. We calculate the coupled cavities' far-field radiation pattern using methods described by Kim et al., ${ }^{11}$ the results are shown in Figs. 2(b) and 2(c). While the PhC cavities' mode profile and $Q$ hardly change with the presence of the substrate under the suspended $\mathrm{PhC}$ slab, the far-field pattern is noticeably affected, making the reflectivity and distance of the subtrate an important design parameter. Single L3 or L5 cavities radiate light with poor directionality. Most of Design A's emis-
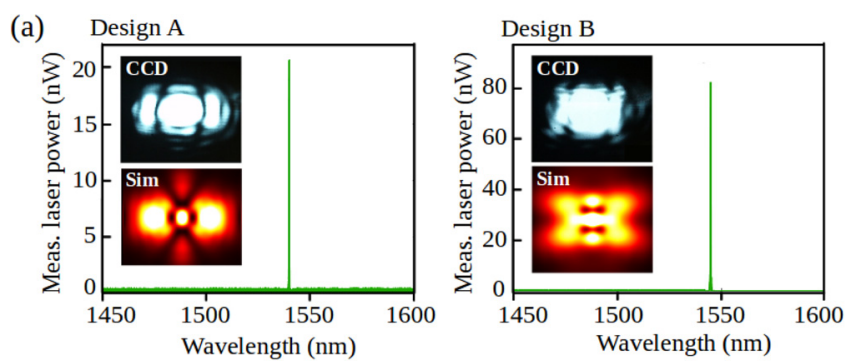

(b)
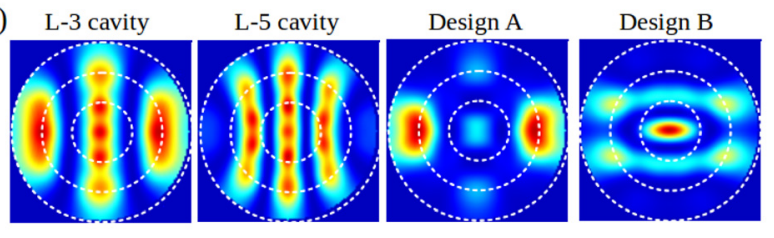

(c)
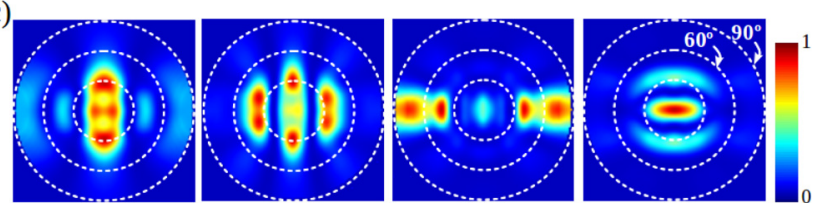

FIG. 2. (Color online) (a) Lasing spectra of coupled-cavity lasers, insets show CCD camera image of the lasing device and its corresponding simulation results. Simulation of far-field emission patterns for single L3, L5, and coupled-cavity lasers: (b) suspended $\mathrm{PhC}$ slab with no substrate and (c) $\mathrm{PhC}$ slab suspended $1160 \mathrm{~nm}$ above the InP substrate. Dashed white circles indicate $30^{\circ}, 60^{\circ}$, and $90^{\circ}$ from surface normal. sion is at $\pm 60^{\circ}$ in the $x$-direction, making free-space light collection difficult. Under the comparison, Design B has much enhanced emission directionality along the laser's surface normal, suggesting efficient free-space coupling. For Design B of coupled-cavity laser suspended $1.16 \mu \mathrm{m}$ above the InP substrate as is our case, the emitted light has a Gaussian-like dominant center peak. The center emission lobe represents $\sim 40 \%$ of the light emitted from the laser's top surface and has a full width at half maximum (FWHM) beam divergence of $20^{\circ}$ from surface normal in the $x$-direction and $8^{\circ}$ in the $y$-direction.

InAsP/InP QWs have the potential advantage of higher temperature and lower threshold operation, due to their greater band-edge offset and thus better carrier confinement as well as expected weaker surface recombination compared with the commonly used InGaAsP/InP QW slabs. ${ }^{15}$ However, this material system has not received much attention for $1.55 \mu \mathrm{m}$ wavelength-scale lasers. We proceed to characterize our best performing device using Design B to provide more insight into device performance and material properties. First, we analyze the measured light-in versus light-out $(L-L)$ curve using the 3-level rate equations ${ }^{6,16}$

$$
\begin{gathered}
\frac{d N}{d t}=\frac{\eta L_{i n}}{\hbar \omega_{p} V_{a}}-v_{g} g(N) P-N\left(\frac{1-\beta+F_{p} \beta}{\tau_{s p}}\right)-A N-C N^{3} \\
\frac{d P}{d t}=-\frac{P}{\tau_{p h}}+\Gamma F_{p} \beta \frac{N}{\tau_{s p}}+\Gamma v_{g} g(N) P,
\end{gathered}
$$

where gain is approximated by $g(N)=g_{0} \ln \left(N / N_{t r}\right), \omega_{p}$ is the pump frequency, and $\tau_{p h}$ is the photon lifetime calculated from cold cavity $Q$. The ratio of pump power that generates carriers in the active region $\eta$, the transparency carrier density $N_{t r}, \beta$, the spontaneous recombination lifetime $\tau_{s p}$, and Purcell factor $F_{p}$ are variables in the fit, from which the carrier density $N$, the photon density $P$, the pump power $L_{i n}$, and the device output power $L_{\text {out }}$ are calculated. The independent variables and their respective values are summarized in Table I. The effective pump power is evaluated from the overlap efficiency of the pump laser light with the photonic crystal cavity and the absorption efficiency in the InAsP/InP slab. ${ }^{6,8}$

We fit the $L-L$ curve to a range of likely gain coefficient $g_{0}=1000-2000 \mathrm{~cm}^{-1}$. The rate equation fit results are consistent despite changes in $g_{0}$. Lasing threshold is $14.6 \mu \mathrm{W}$, very low for a 4-QW device with an effective mode volume $V_{\text {eff }}$ of $1.9(\lambda / n)^{3}$. Transparency carrier density is about $1 \times 10^{18} \mathrm{~cm}^{-3} \cdot \tau_{s p}$ is $4-7 \mathrm{~ns}$. Purcell factor $F_{p}$ is between 1 and 1.5 due to InP/InAsP QW material's homogeneous broadening at room temperature and the coupled-cavity's

TABLE I. Material and device parameters used in rate equation analysis (Ref. 16).

\begin{tabular}{lc}
\hline \hline Parameter & Value \\
\hline Effective modal volume $V_{\text {eff }}$ & $0.223 \mu \mathrm{m}^{3}$ \\
Confinement factor $\Gamma$ & 0.0966 \\
Surface recombination velocity $v_{s}$ & $10^{4} \mathrm{~cm} / \mathrm{s}$ \\
Propagation distance to suface $d_{a}$ & $2 \times 10^{-5}$ \\
Surface recombination rate $A=v_{s} / d_{a}$ & $5 \times 10^{8} / \mathrm{s}$ \\
Auger recombination rate $C$ & $4.25 \times 10^{-29} \mathrm{~cm}^{6} / \mathrm{s}$ \\
\hline
\end{tabular}


(a)

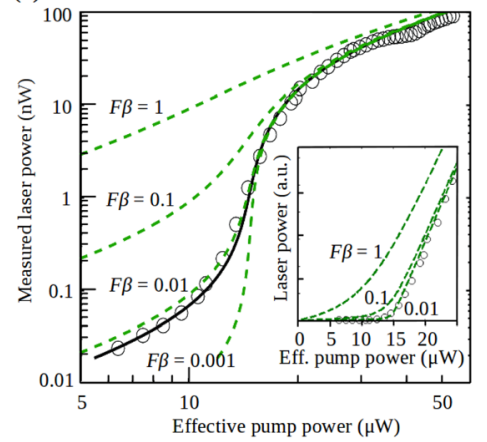

(b)

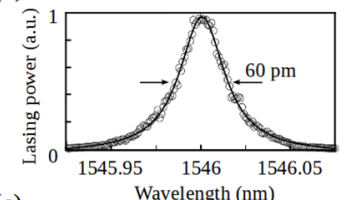

(c)

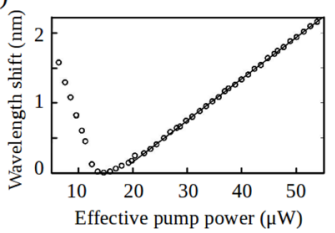

FIG. 3. (Color online) Design B device measurements: (a) rate equation fitted $L-L$ measurement on $\log -\log$ scale, circles represent measured data, solid black line is the fit, calculated $F_{p} \beta=1,0.1,0.01$, and $0.001 . L-L$ curves using the fit data are in green dashed lines. Inset shows a zoom-in at the threshold in linear scale. (b) Measured linewidth, solid line shows the Lorentzian fit. (c) Measured wavelength shift versus effective pump power, solid line is the above-threshold $d \lambda / d W$ fit.

somewhat large $V_{\text {eff. }}{ }^{6} F_{p} \beta \approx 0.01$. The fit with $g_{0}=1500$ $\mathrm{cm}^{-1}$ is shown in Fig. 3(a). In measuring the coupled-cavity laser's $L-L$ curve, the device was pumped to over $100 \mathrm{nW}$ as recorded by the OSA, and we expect it to operate stably at even higher powers. The lasing peak is easily $\geq 30 \mathrm{~dB}$ above noise level in our measurement. We estimate that about $1 \%$ of the laser's output power is coupled to the OSA due to loss through optical elements in the optical path from the device to the OSA. Thus, the coupled-cavity laser's output power is on the order of $10 \mu \mathrm{W}$.

Lasers with single mode CW operation can achieve stable emission with a narrow linewidth, which directly influences the device's performance in applications such as spectroscopy and coherent optical communication. We measure the linewidth when the device is pumped at 3 times the threshold pump power. The data is shown in Fig. 3(b) and is fitted to a Lorentzian. We find that the FWHM lasing linewidth is $60 \mathrm{pm}$. Measurement accuracy is limited by the OSA's minimum achievable resolution of $15 \mathrm{pm}$. Lasing frequency shifts with increasing pump intensity as the combined result of blue shift due to carrier plasma effect with increased free carrier density in the cavity and red shift due to device heating. Measured lasing wavelength shift versus effective pump power is shown in Fig. 3(c), clearly showing

free carrier dominant blue-shift below threshold and heating dominant red-shift above threshold, which measures to be $0.059 \mathrm{~nm} / \mu \mathrm{W}$.

In summary, we have demonstrated RT CW operation in coupled-cavity photonic crystal lasers with a very low threshold power of $14.6 \mu \mathrm{W}$, a linewidth of $60 \mathrm{pm}$, and enhanced far-field radiation directionality. The larger area coupled-cavity will be advantageous in applications where both the laser output power and the threshold are of concern.

The authors would like to acknowledge support from the Defense Advanced Research Projects Agency under the Nanoscale Architecture for Coherent Hyperoptical Sources programme under Grant No. \#W911NF-07-1-0277 and from the National Science Foundation through NSF CIAN ERC under Grant No. \#EEC-0812072. J. G. would like to acknowledge support from NSF Research Experience for Teachers (RET) program through NSF CIAN ERC. P. A. P. would like to acknowledge financial support from Spanish MICINN and CAM through grants NANINPHO-QD (TEC2008- 06756C03-01/03), CAM2010 Q\&C Light (S2009ESP-1503) and Consolider-Ingenio 2010 QOIT (CSD2006-0019).

${ }^{1}$ D. A. B. Miller, Proc. IEEE 97, 1166 (2009).

${ }^{2}$ M. Lončar, A. Scherer, and Y. M. Qiu, Appl. Phys. Lett. 82, 4648 (2003).

${ }^{3}$ S.-H. Kim, J.-H. Choi, S.-K. Lee, S.-H. Kim, S.-M. Yang, Y.-H. Lee, C. Seessal, P. Regrency, and P. Viktorovitch, Opt. Express 16, 6515 (2008).

${ }^{4}$ Y. Yamamoto, S. Machida, and G. Björk, Phys. Rev. A 44, 657 (1991).

${ }^{5}$ H. Altug, D. Englund, and J. Vučković, Nature Phys. 2, 484 (2006).

${ }^{6}$ K. Nozaki, S. Kita, and T. Baba, Opt. Express 15, 7506 (2007).

${ }^{7}$ M. Nomura, S. Iwamoto, K. Watanabe, N. Kumagai, Y. Nakata, S. Ishida, and Y. Arakawa, Opt. Express 14, 6308 (2006).

${ }^{8}$ L. J. Martinez, B. Aln, I. Prieto, D. Fuster, L. Gonzlez, Y. Gonzlez, M. L. Dotor, and P. A. Postigo, Opt. Express 17, 14993 (2009).

${ }^{9}$ K. Srinivasan, M. Borselli, O. Painter, A. Stintz, and S. Krishna, Opt. Express 14, 1094 (2006).

${ }^{10}$ K. Nozaki, H. Watanabe, and T. Baba, Appl. Phys. Lett. 92, 021108 (2008).

${ }^{11}$ S.-H. Kim, S.-K. Kim, and Y.-H. Lee, Phys. Rev. B 73, 235117 (2006).

${ }^{12}$ H. Altug and J. Vučković, Opt. Express 13, 8819 (2005).

${ }^{13}$ A. R. Alija, L. J. Martínez, P. A. Postigo, C. Seassal, and P. Viktorovitch, Appl. Phys. Lett. 89, 101102 (2006).

${ }^{14}$ N. -V.-Q. Tran, S. Combrié, and A. D. Rossi, Phys. Rev. B 79, 041101(R) (2009).

${ }^{15}$ G. Dagnall, J.-J. Shen, T.-H. Kim, R. A. Metzger, A. S. Brown, and S. R. Stock, J. Electron. Mater. 28, 933 (1999).

${ }^{16}$ L. A. Coldren and S. W. Corzine, Diode Lasers and Photonic Integrated Circuits (Wiley, New York, 1995). 